\title{
A mechanical approach to realize reflexive omnidirectional bending motion for pneumatic continuum robots
}

\author{
Eri Takane ${ }^{*}$, Kenjiro Tadakuma, Tomonari Yamamoto, Masashi Konyo and Satoshi Tadokoro
}

\begin{abstract}
A mechanism that allows a robotic arm to quickly grip various forms of objects at disaster sites will enhance the mobility of rescue robots by keeping their bodies stable and maintaining manipulability for target objects, such as debris. Such a mechanism requires the ability to quickly and omnidirectionally change arm postures toward the target and hold it in a stable manner. Continuum robots are expected to provide this functionality. Conventional continuum robots realize the function of changing arm postures and grasping objects by controlling pneumatic actuators with multiple air chambers arranged in parallel. However, conventional robots cannot be applied to potential disaster sites filled with flammable gases, gasoline, or high radiation levels because they require electronic components (e.g., solenoid valves, and sensors) to control air pressures. This study proposes a unique approach to realize reflexive omnidirectional bending motion using only mechanical components without any electrical devices. The proposed system realizes a reflexive motion to bend the arm in the target's direction by detecting a contact location using a mechanical reactive system. The proposed simple mechanism has the advantages of high durability and easy implementation. This paper aims to confirm the proposed concept by prototyping a drive mechanism coupled with contact detection and bending motion using mechanical port valves. We report the design concept and development of this prototype. The fundamental characteristics and feasibility of the proposed mechanism are experimentally confirmed. First, a prototype is developed using a mathematical model. Its performance in the bending and omnidirectional motions is evaluated. The results show that the model has a margin of $-4.9 \%$ error in the bending angle and $-7.4 \%$ error in the central curvature compared with the experimental values. We also confirm that using a higher pressure could realize a smaller radius of curvature and reduce an unnecessary twisting motion. We also tested a second prototype to confirm the grasping motion and force by changing the applied pressures. The influence of the bending direction was then evaluated. We confirm that a higher pressure generated a larger grasping force. The prototype can omnidirectionally produce approximately the same forces although the generated forces depend on the number of air chambers excited by the contact pads. Subsequently, we experimentally confirm the influence of gravity. The test shows that the effect of own weight greatly influences the posture after the object is in contact. This effect should not be ignored. Furthermore, the curve became sufficiently large when its contact pad is pressed. This result experimentally proved that self-holding is possible. The experimental results show the potential of the proposed mechanism.
\end{abstract}

Keywords: Omnidirectional bending mechanism, Continuum robots, Reflexive motion

\footnotetext{
*Correspondence: takane@rm.is.tohoku.ac.jp

Graduate School of Information Sciences Applied Information Sciences

Information and Applied Technology Human-Robot Informatics, Tohoku

University, Sendai, Miyagi, Japan
} 


\section{Background}

Mechanisms that allow gripping of objects of various forms, sizes, fragilities, and sensitivities are highly desirable at disaster sites. For example, a robotic arm should enhance manipulability in picking up a target object, such as a piece of debris, and should augment the mobility of rescue robots by keeping their bodies stable, such as at the end-effector of continuum robots, to perform brachiation inspired by animal movements. Such mechanisms require the ability to change the arm's posture quickly and omnidirectionally toward the target to hold it stable.

Continuum robots are expected to realize the abovementioned functionalities, and have already been introduced for rescue operations [1-6]. Many conventional studies report on gripper mechanisms that can accommodate various forms of objects via deformation functionality using a differential coupled mechanism [7], morphing structure $[8]$, particle jamming effects $[9,10]$, and flexible links $[11,12]$.

Most conventional continuum robots particularly realize the function of changing arm posture and grasping objects by controlling pneumatic actuators with multiple air chambers arranged in parallel [13-17]. The pneumatic drive, which is the beginning of it, was an explosionproof system for coal mine machinery. It was also used as an explosion-proof measure for the gas current. Therefore, systems without electronic components (e.g., solenoid valves, and sensors) to control air pressures can be applied in potential disaster sites filled with flammable gases, gasoline, or high radiation levels.

We propose herein a unique approach to realize reflexive omnidirectional bending motion by using only mechanical components without any electrical devices. The system proposed realizes a reflexive motion to bend the arm toward the target direction by detecting a contact location using a mechanical reactive system. The proposed simple mechanism has the advantages of high durability and easy implementation. This paper aims to confirm the concept and feasibility of the proposed reflexive bending mechanism coupled with contact detection using prototypes and experimentally report the fundamental characteristics. First, we discuss the possible design concepts for the omnidirectional bending motion and introduce a new design to realize the reflexive motion by means of a coupled drive mechanism with touch detection using mechanical port valves. Second, we develop a first prototype with a mathematical model and evaluate its performance in the bending and omnidirectional motions. Third, we investigate the grasping force generated and the influence of the bending direction and gravity by developing a second prototype that can grasp a cylindrical bar by changing the applied pressures.

\section{Methods}

We consider herein an all-mechanical approach for a reflexive omnidirectional bending mechanism. The bending arms for the bending motion toward the direction of a contacted object after detection of the contact with the object can be classified based on whether they are holonomic. As shown in Fig. 1a, an omnidirectional bending arm mechanism can be realized as a non-holonomic motion by rotating a configuration that can bend in one direction. However, the time between the contact with the object and grasping is increased because a rotational motion occurs after contact has been made with an object. Therefore, we employed a holonomic system that directly bends in the object direction. The bending mechanisms for the holonomic systems have been extensively studied (e.g., Refs. [13-17]). A discussion of the designs used to realize a holonomic bending function is presented on the sections that follow along with the design proposed in this paper.

We present a concrete method to approach an allmechanical system that reflexively bends in the direction of a contacted object. A coupled drive mechanism with touch detection using a mechanical port valve is used herein to realize the proposed mechanism. The coupled drive mechanism with this touch detection is the basic principle of a reflexive omnidirectional bending mechanism (Fig. 2). Air flows into the bellows on the opposite side of the device (bellows "A") when pad "a" is pushed by an object. In this way, the configuration can bend around objects. Furthermore, the use of more than one air chamber allows the bending to be realized (Fig. 2). Note that the cross-sectional shape is not required to be circular.

Figure 3 presents the basic principle of mechanical touch sensing for self-bending motion using mechanical port valves. The figure illustrates a cross-sectional view of the structure shown in Fig. 2. The bellows are usually opened to the atmosphere through a mechanical port valve as shown in Fig. 3a. In addition, the contact pad

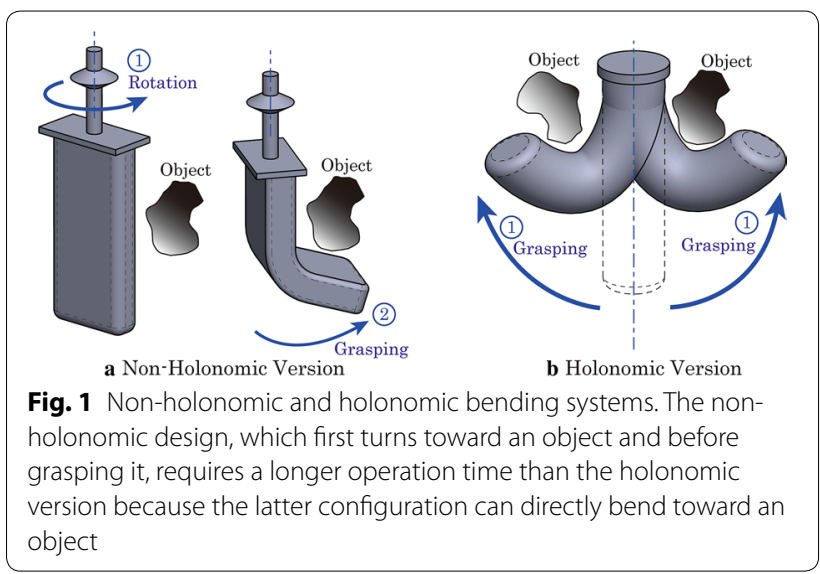




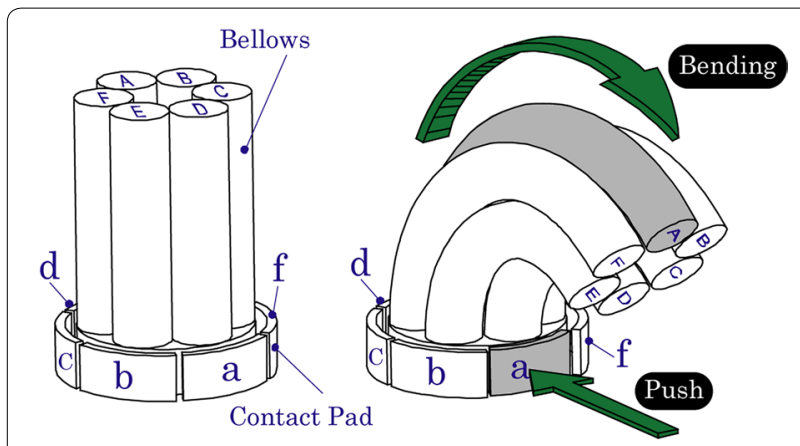

Fig. 2 Basic design principle of the reflexive omnidirectional bending mechanism with passive touch detection. Bellows $A$ receives air when contact pad " $a$ " is pushed inward through contact with an object. This configuration can then bend in the direction of the object

was equipped with mechanical port valves connected to the bellows on the opposite side of the device. Therefore, the bellows on the opposite side of the device connect to the pneumatic tank and extend, as shown in Fig. 3b, when an object pushes on the contact pad. This mechanism realizes reflexive omnidirectional bending. Figure 4 shows the design and prototype of the proposed reflexive omnidirectional bending mechanism with coupled drive mechanism and touch detection. Table 1 presents the prototype specifications. The prototype was designed with six sets of valves and bellows. The use of more sets corresponds to the increased directional resolution. However, increasing the resolution is necessary to examine the design stage because it leads to an increase in the part count, weight, and size. The bending motion is modeled in this section using this prototype. We described the model evaluation and omnidirectional motion in the next section.

The maximum bending position after contact is modeled to determine the expected size of an object that the mechanism can grasp at the design stage. The maximum bending position can be measured by obtaining the radius of curvature and the bending angle for each pressure if the bending motion of the one-unit system is assumed to describe an arc. We assumed that the mechanism for this analysis exhibits zero twisting; hence, it was modeled in $2 \mathrm{D}$ and without gravity. Figure 5 shows the bending motion modeled on the $2 \mathrm{D}$ cross-section. Accordingly, $d$ is the distance between bellows A (outside bellows) and $\mathrm{D}$ (inside bellows); $R$ is the maximum radius of curvature of bellows $\mathrm{D}$; and $\theta$ is the bending angle. In addition, the minimum length of each bellows is labeled as $L_{1}$, while $L_{1}+\alpha$ corresponds to the length of bellows $\mathrm{D}$ during bending. Bellows $\mathrm{D}$ is shortened at the maximum bending position, and is then extended by $\alpha . L_{2}$ is the maximum length of each bellows, while $L_{2}$ at each

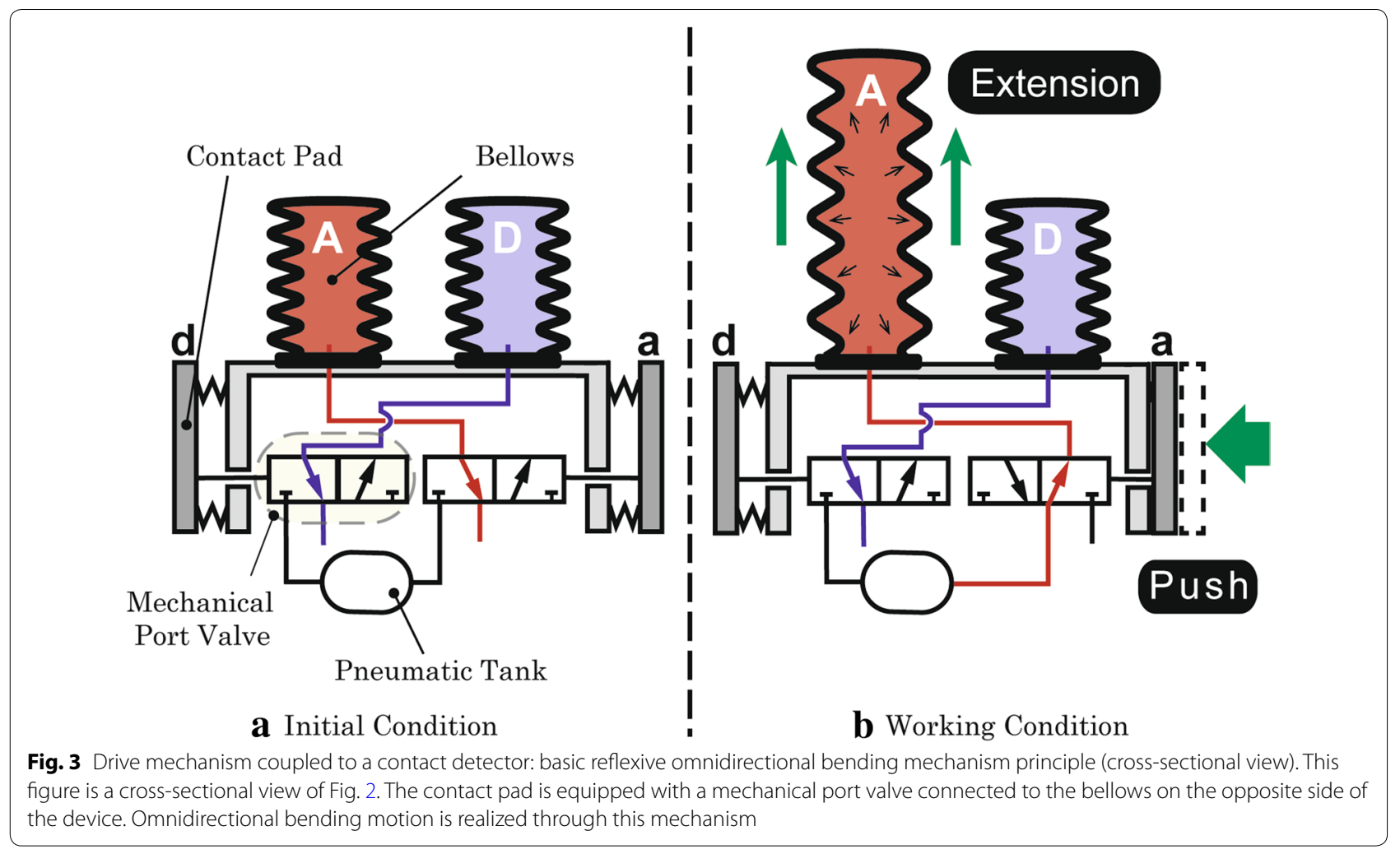




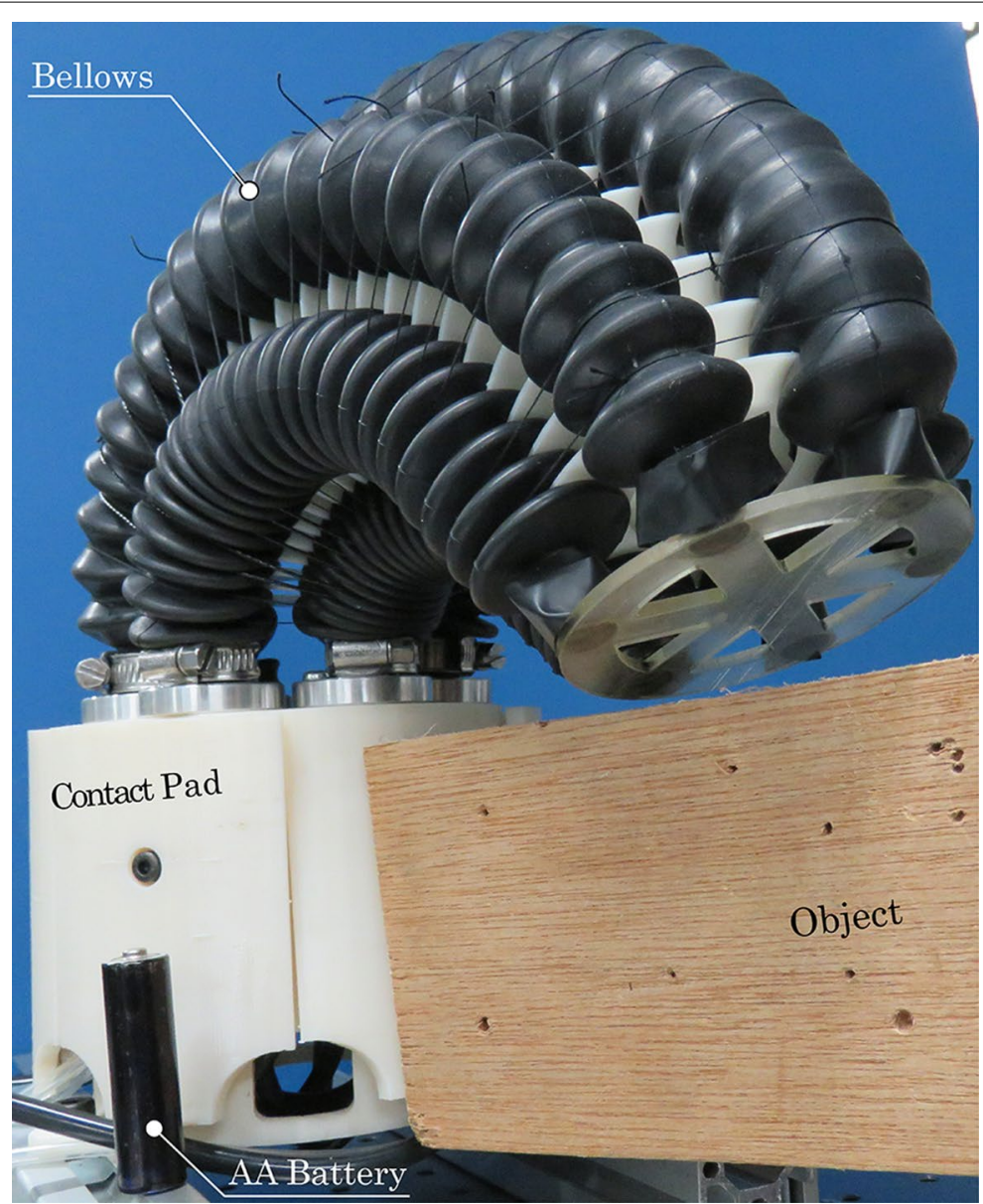

Fig. 4 Photograph of the first prototype model exterior. The prototype can bend in the direction of a contacted object. A AA battery is provided to indicate scale

\section{Table 1 First prototype model specifications}

\begin{tabular}{ll}
\hline One bellows & 50 \\
Hardness & $20 / 35$ \\
Internal/external diameter (mm) & 195 \\
Natural length (mm) & 90 \\
Minimum length $(\mathrm{mm})$ & 280 \\
Maximum length (mm) & Chloroprene rubber \\
Rubber material & \\
Entire device & 20 \\
Set pressure (kPa) & 1552 \\
Weight (g) & 130 \\
External diameter (mm) & 210 \\
Minimum length (mm) & 390 \\
Maximum length (mm) & 169 \\
Radius of curvature (mm) & \\
Components & MSHRP3 (MISUMI Corporation) \\
Port valve & ITV10J1-211L (SMC Corporation) \\
Regulator & 6 \\
Pipe diameter (mm)
\end{tabular}

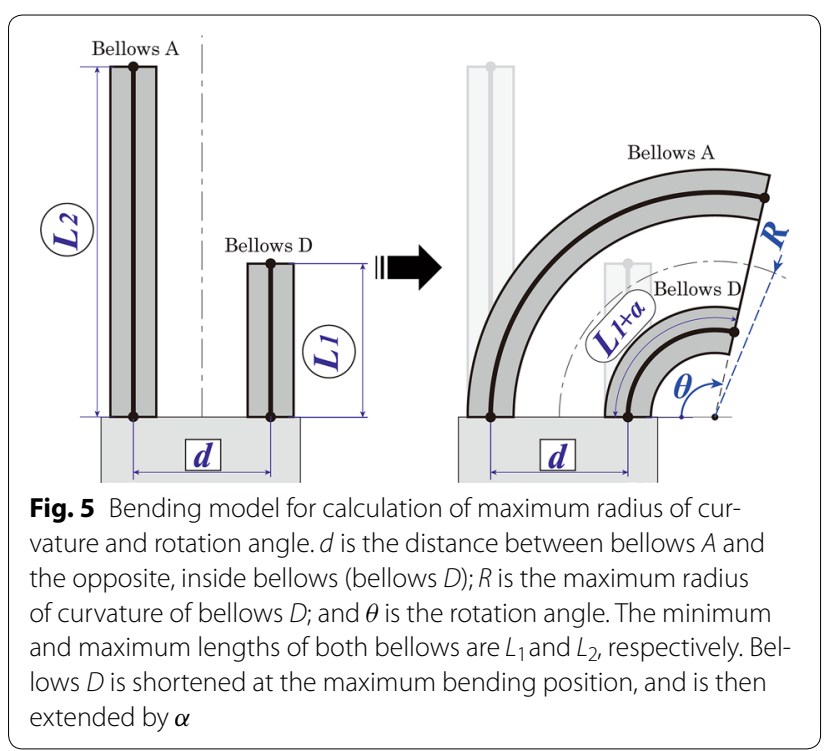


Table 2 First prototype model's maximum length of bellows a for various applied pressures

\begin{tabular}{ll}
\hline Pressure $(\mathbf{k P a})$ & Maximum length $(\mathbf{m m})$ \\
\hline 5 & 320 \\
10 & 345 \\
15 & 375 \\
20 & 385 \\
\hline
\end{tabular}

pressure is already known from the previous experiment. Table 2 shows the maximum length values of this prototype at each pressure for bellows A. Accordingly, $\theta$ and $R$ can be expressed as follows because of the geometric relationship shown in Fig. 5:

$$
\begin{aligned}
& \theta=\frac{L_{2}-\left(L_{1}+\alpha\right)}{d}, \\
& R=\frac{\left(L_{1}+\alpha\right) \cdot d}{L_{2}-\left(L_{1}+\alpha\right)}+\frac{d}{2} .
\end{aligned}
$$

The reflexive omnidirectional bending mechanism can then be extended based on this one unit. This mechanism can grasp larger objects by extending a grasping part. Furthermore, Eqs. (1) and (2) can be used in the extended versions.

The extended bellows grasping part is designed, prototyped, and examined herein. The grasping motion for the applied pressure was particularly confirmed. The grasping force was measured, and the influence of the bending direction was verified.

\section{Results and discussion}

\section{First prototype: posture measurements}

We measured the bending motions using a motion capture system when a contact pad was manually pushed by an experimenter's hand to confirm the basic temporal posture profiles achieved by changing the applied pressures in the chambers Fig. 6 shows the experimental setup. Markers for the motion capture system were attached to the device as shown in the figure. AM02-04N (Fujiwara Sangyo Co.), which had been used in subsequent experiments, was used as the compressor.

The initial state was defined as the attitude inclined to the left side by the prototype's weight (Fig. 6 (1)). The pictures in the series from (2) to (6) in Fig. 6 show examples of the posture profiles when the contact pads are pushed. Figure 6 (6) shows the maximum bending posture, which depends on the applied pressure. The applied pressures in the experiments were $5,10,15$, and $20 \mathrm{kPa}$.

Figure 7 shows the measured temporal posture profiles during the bending motion. These results confirmed that higher applied pressures produced higher speed movements from the initial bending state and larger curvatures.

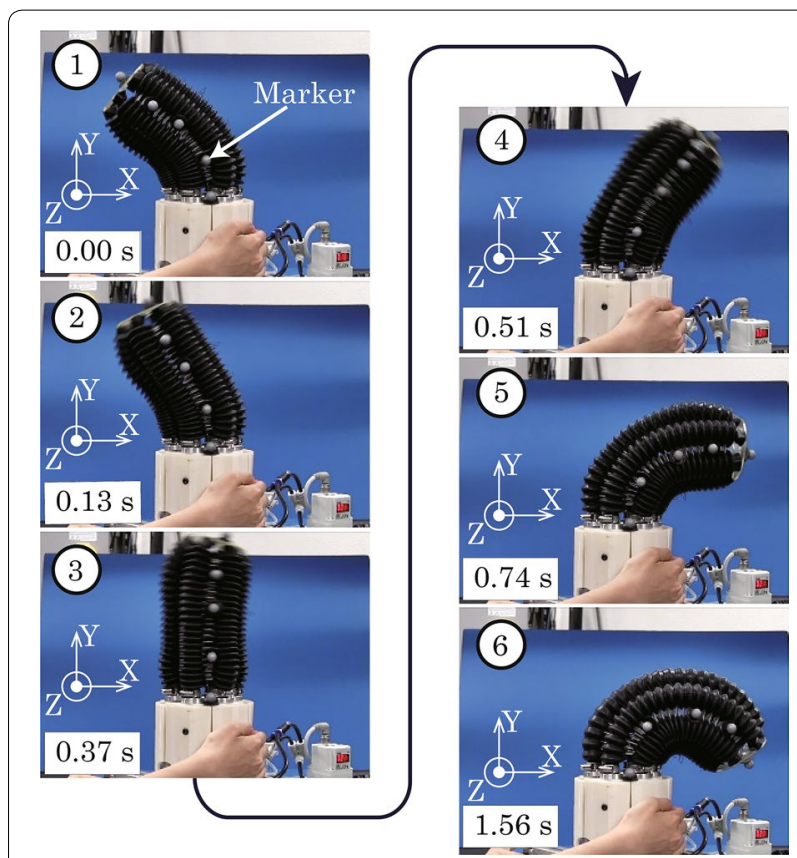

Fig. 6 Motion-Capture Experimental Setup. Five motion capture markers are attached to the prototype to monitor its position

The bending movement slowed down at the final state near the maximum position. The required time to reach the maximum bending position was longer than that at $15 \mathrm{kPa}$ when the pressure was $20 \mathrm{kPa}$. However, the bending position reached a larger curvature. Figure 8 shows a comparison of the posture at the final state for each applied pressure. The figure clearly shows differences in the curvature depending on the applied pressure. The applied pressure also affected the twisting movement in the $\mathrm{z}$-axis. The twist indicates that the track in the contact direction cannot draw a straight line on the xy plane, and the prototype cannot bend out of alignment because of individual differences of the air camber at the time of production and because a deviation occurs. Table 3 shows the RMS of the tip position errors along the $\mathrm{z}$-axis for each applied pressure. The results showed that higher pressures could generate stable bending motions with smaller errors along the $\mathrm{z}$-axis. These errors resulted from the unnecessary twisting motions of the prototype, which may have been caused by the higher stiffness caused by the higher pressures in the chambers in their parallel configuration.

We then compared the theoretical posture calculated by the mathematical model described in the method section to identify the model parameters and confirm the validity of the proposed model. The model parameters include $d$ and $L_{1}$ and $\alpha$, which corresponded to $90 \mathrm{~mm}$ and $90 \mathrm{~mm}$, respectively. Accordingly, $\alpha$ was identified to the value closest to the experimental value from Eqs. (1) and (2). Consequently, we identified that $\alpha$ was $27.3 \mathrm{~mm}$. 


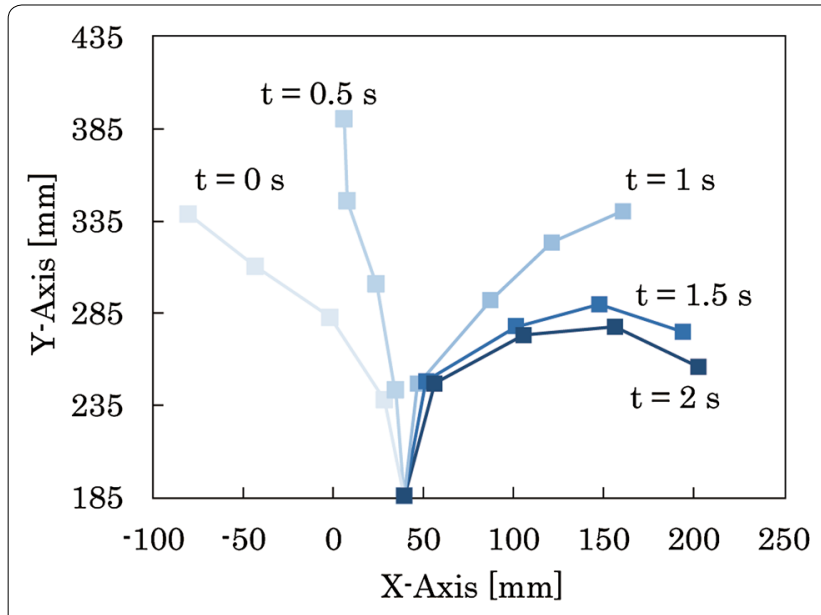

a Bending Positions at Various Times $(\mathrm{t})$ for $5 \mathrm{kPa}$ Internal Pressure

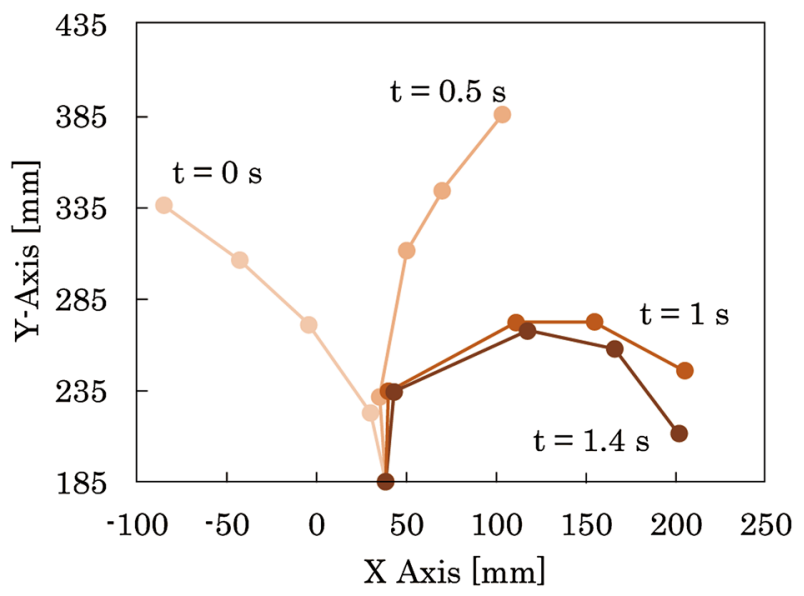

c Bending Positions at Various Times $(\mathrm{t})$

for $15 \mathrm{kPa}$ Internal Pressure

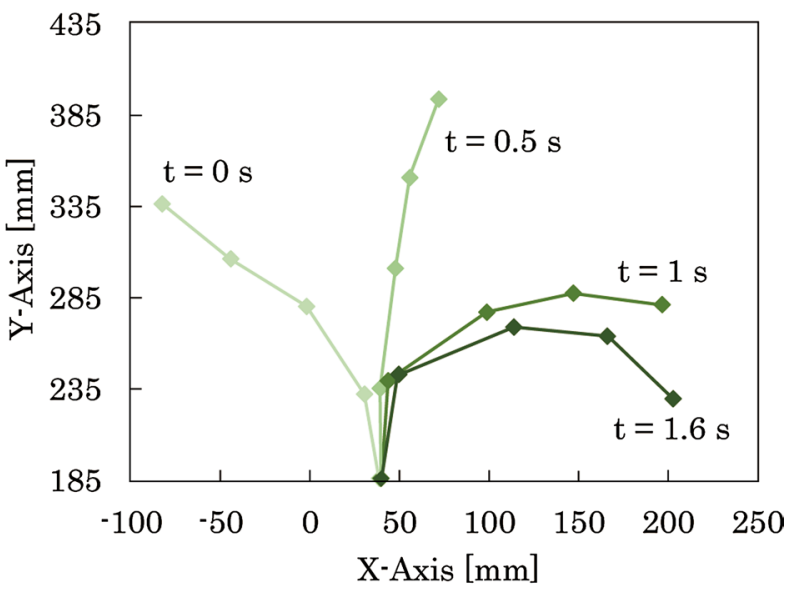

b Bending Positions at Various Times ( $\mathrm{t}$ ) for $10 \mathrm{kPa}$ Internal Pressure

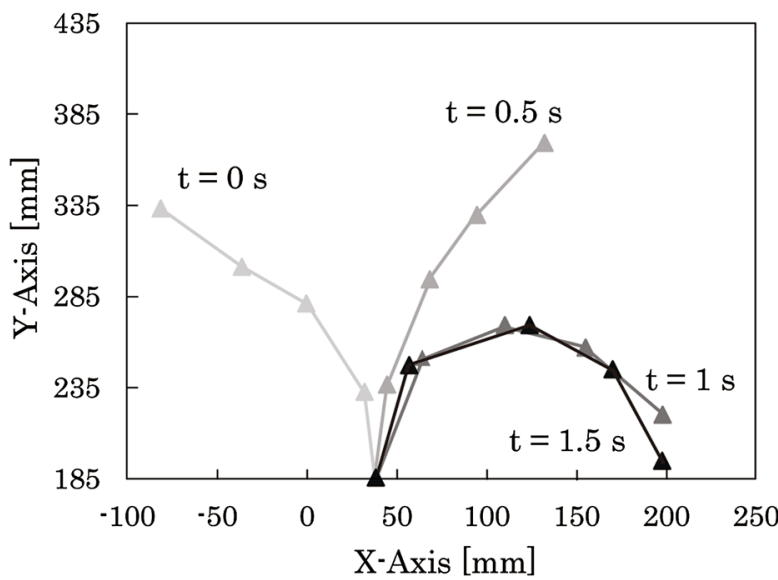

d Bending Positions at Various Times ( $t$ ) for $20 \mathrm{kPa}$ Internal Pressure

Fig. 7 Temporal posture profiles. a Under $5 \mathrm{kPa}$ pressure, aA period of $2 \mathrm{~s}$ was required for maximum bending under $5 \mathrm{kPa}$ pressure. $\mathbf{b} \mathrm{Under} 10 \mathrm{kPa}$ pressure, Aa period of $1.6 \mathrm{~s}$ was required for maximum bending under $10 \mathrm{kPa}$ pressure. $\mathbf{c}$ Under $15 \mathrm{kPa}$ pressure, Aa period of $1.4 \mathrm{~s}$ was required for maximum bending under $15 \mathrm{kPa}$ pressure. d Under $20 \mathrm{kPa}$ pressure, aA period of $1.5 \mathrm{~s}$ was required for maximum bending under $20 \mathrm{kPa}$ pressure

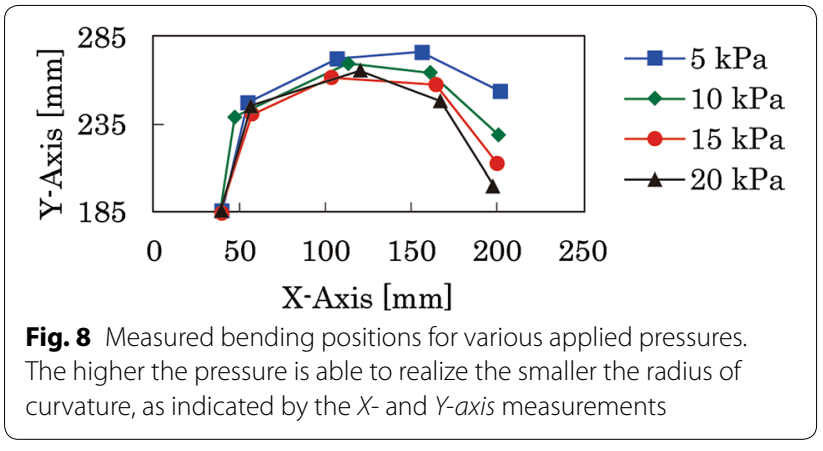

Therefore, the averaged error of all the pressures was minimized. The maximum error of $\theta$ was $-4.9 \%$ in the case of $10 \mathrm{kPa}$, and that of $R$ was $-7.4 \%$ in the case of $5 \mathrm{kPa}$. The measured postures were the same as those shown in Fig. 8. Figures $9 \mathrm{a}, \mathrm{b}$ show a comparison between the experimental results and the theoretical values of the bending angle $\theta$ and the radius of curvature $R$, respectively. The experimental results showed the quantitative characteristics of the postures in the final state when the applied pressures were changed. These results agreed well with the tendencies, that is, higher pressures produced larger $\theta$ and smaller 
Table 3 The RMS of tip position errors along the Z-axis for various applied pressures

\begin{tabular}{ll}
\hline Pressure (kPa) & $\begin{array}{l}\text { RMS of tip } \\
\text { position errors }\end{array}$ \\
\hline 5 & 9.78 \\
10 & 7.88 \\
15 & 6.65 \\
20 & 4.30 \\
\hline
\end{tabular}

$R$, as described earlier. We confirmed that the theoretical values agreed well with the experimental results.

\section{First prototype: omnidirectional reflexive motion and grasping test}

The proposed mechanical configuration is expected to reflexively generate the bending motion in the direction of contact. We conducted an experiment, in which the mechanism was pushed from multiple directions, to confirm the reflexive motion. All contact pads in the experiment were pressed with a wooden stick, thereby continuously changing the direction. The reflexive movement and bending directions were observed. Figure 10 shows an example of the reflexive bending movement when three different contact pads (i.e., \#1, \#2, and \#3) were pressed sequentially. The series of pictures demonstrated that the proposed configuration could generate a reflexive action to directly change the bending direction from the first contact point to the next contact point. We confirmed that the prototype could bend in all other directions.

We then conducted a brief test to confirm the gripping capability of the first prototype. Figure 11 shows the gripping motion when the target object was a urethane sponge with a width of $60 \mathrm{~mm}$. The pictures showed a limitation of the first system in holding large objects caused by the insufficient arm length. From this observation, we have confirmed that long arm length is necessary in grasping an object by wrapping around it. We extended the length in the following section to verify the grasping capability.

\section{Second prototype extended grasping part: configuration} We developed a longer prototype by extending the length of the bellows grasping part twice. Figure 12 shows the prototype developed when successfully grasping a transparent object. Table 4 presents the prototype specifications.

The bellows parts cannot directly detect the contact events in this configuration because of the large distance between the contact pads and the grasping parts. We focused herein on the verification of our fundamental concept of the reflexive omnidirectional bending motion using an all-mechanical system. We did not discuss a sensing method for the bellows. In the future, we will consider rearranging multiple contact detection parts in a discrete manner or introducing a continuous sensing structure to combine the detection parts with the grasping parts.

\section{Second prototype extended grasping part: grasping operation}

We confirmed the grasping capability of the longer prototype by conducting a preliminary grasping test to prove its ability to stably hold an object and release it by selfcontact motion (Fig. 13). The target object was a PET bottle with a $60 \mathrm{~mm}$ diameter, $200 \mathrm{~mm}$ length, and $43 \mathrm{~g}$ weight. The contact pads were pressed separately from the target object by a plastic pipe because of the sensing limitation described earlier.

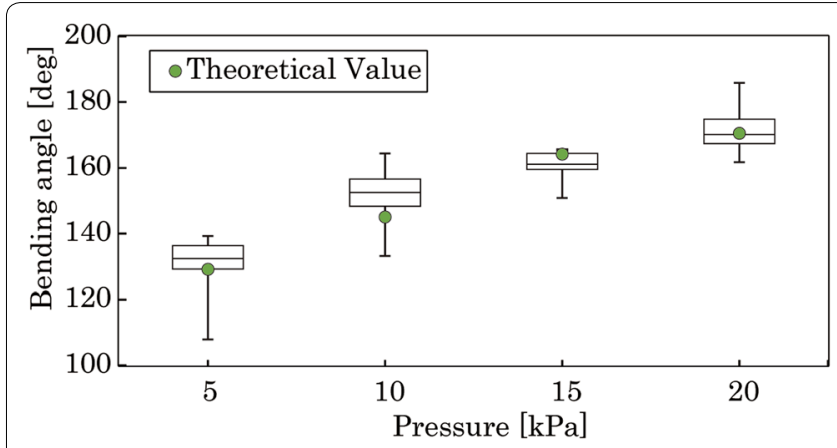

a Experimental and Theoretical Results for Relationship between Pressure and Rotation Angle $\theta$

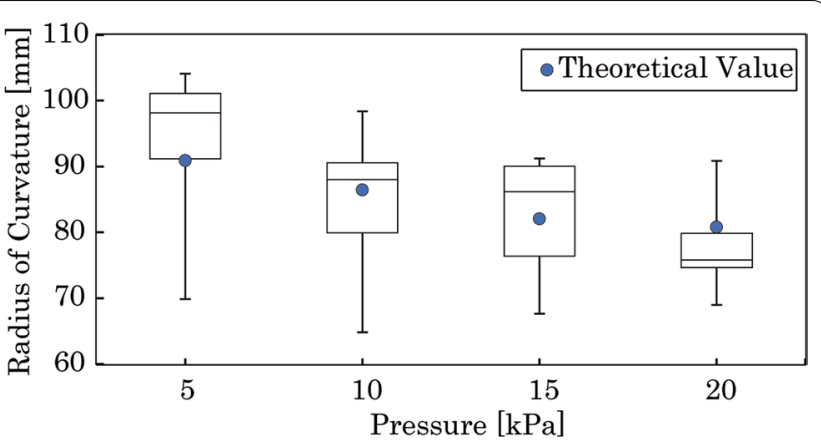

b Experimental and Theoretical Results for Relationship between Pressure and Central Curvature $\mathrm{R}$

Fig. 9 Experimental and theoretical results for the relationship between pressure and rotation angle. $\mathbf{a}$ Changes in $\theta$ in response to changes in the applied pressure are shown. A roughly linear relationship is established. $\mathbf{b}$ Changes in $R$ in response to changes in the applied pressure are shown. A roughly linear relationship is established 


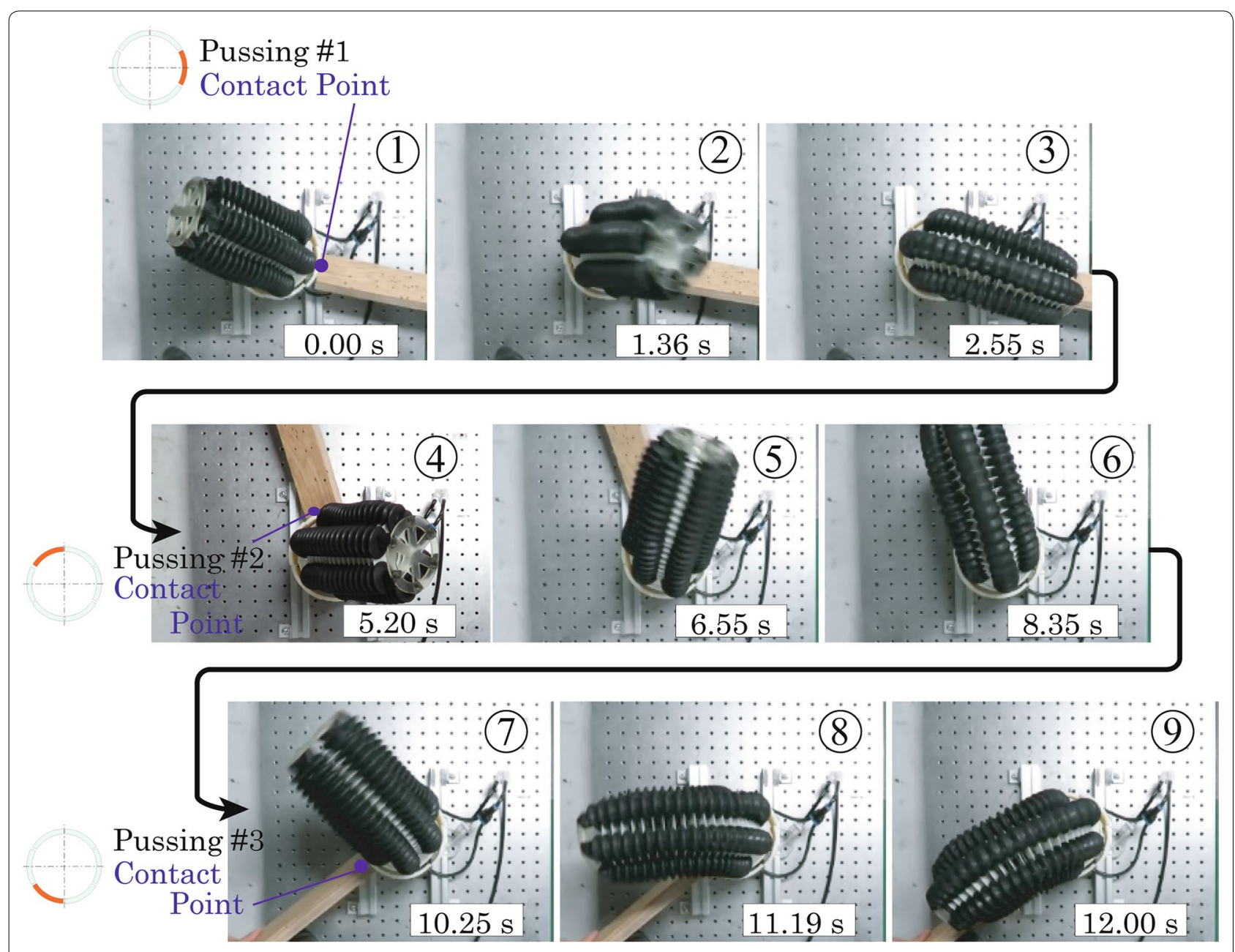

Fig. 10 Omnidirectional bending motion (top view). The photographs confirm that this configuration can bend in an omnidirectional manner relative to the contact point. An additional movie file shows this in more detail (see Additional file 1)

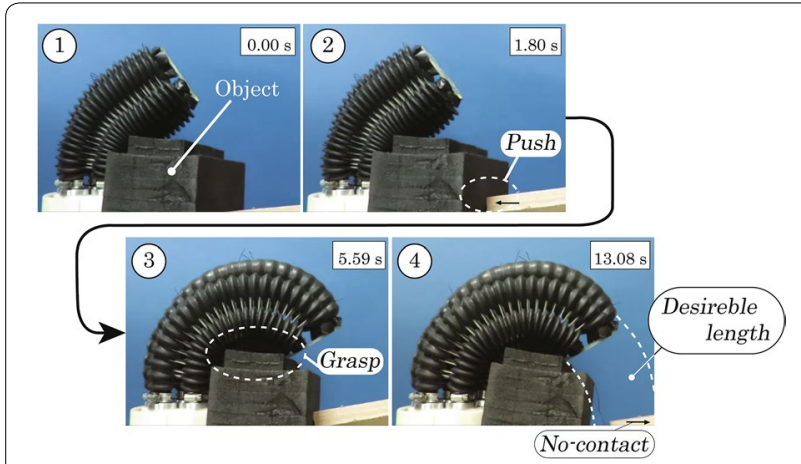

Fig. 11 Grasping motion of the first prototype. It can be seen that the first prototype has insufficient length to grasp the object
The experimental result showed that the longer prototype has the potential to stably grasp and release an object.

\section{Second prototype extended grasping part: grasping force profile measurements}

We measured the temporal force profiles during a grasp by changing the applied pressures to investigate the transient response of the generated force. Figure 14 shows the experimental setup for the measurement. The target object was a cylinder made of polystyrene foam with a $50 \mathrm{~mm}$ diameter, $400 \mathrm{~mm}$ length, and $20 \mathrm{~g}$ weight. The generated forces were measured by a force sensor 


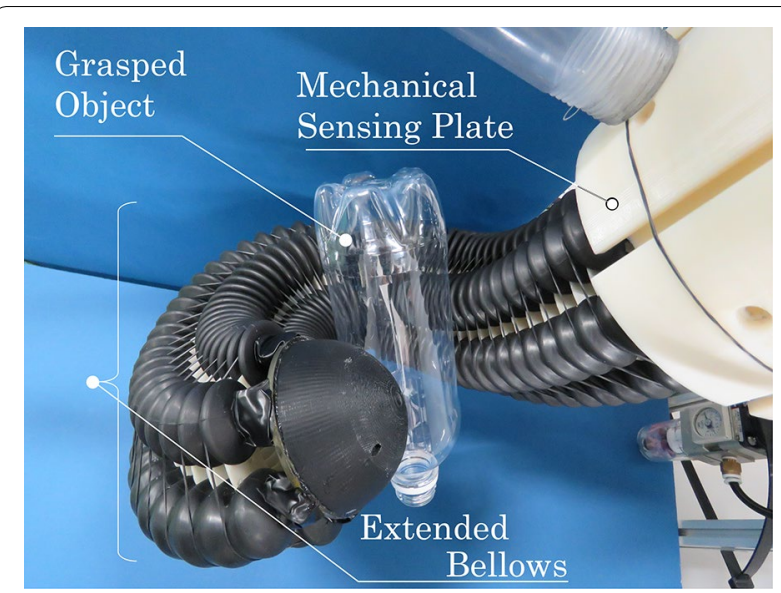

Fig. 12 Photograph of the second prototype model. The second prototype is equipped to grasp an object. An additional movie file shows this in more detail (see Additional file 2)

Table 4 Second prototype model specifications

\begin{tabular}{ll}
\hline $\begin{array}{l}\text { One bellows } \\
\text { Hardness }\end{array}$ & 50 \\
Internal/external diameter $(\mathrm{mm})$ & $20 / 35$ \\
Natural length $(\mathrm{mm})$ & 195 \\
Minimum length $(\mathrm{mm})$ & 90 \\
Maximum length $(\mathrm{mm})$ & 280 \\
Rubber material & Chloroprene rubber \\
Entire mechanism & \\
Weight $(\mathrm{kg})$ & 4.9 \\
External diameter $(\mathrm{mm})$ & 130 \\
Minimum length $(\mathrm{mm})$ & 390 \\
Maximum length $(\mathrm{mm})$ & 680 \\
Components & \\
Port valve & MSHRP3 (MISUMI Corporation) \\
Regulator & ITV10J1-211L (SMC Corporation) \\
Pipe diameter $(\mathrm{mm})$ & 6 \\
\hline
\end{tabular}

(FSR406, Interlink Electronics, Inc.) covering the cylinder surface.

The target object was kept as level as possible by the tester and passed to the prototype under the initial conditions. The prototype then grasped the object for $10 \mathrm{~s}$, and released it after. This process was conducted for pressures of 10, 20, and $30 \mathrm{kPa}$. Figure 15 shows the corresponding results. The grasping force for a $10 \mathrm{kPa}$ pressure at the steady state was $0.50 \mathrm{~N}$, while that for a $20 \mathrm{kPa}$ pressure was $1.4 \mathrm{~N}$. In addition, a force of more than $2.0 \mathrm{~N}$ was obtained when the applied pressure was $30 \mathrm{kPa}$. However, the force value did not reach a steady state within $10 \mathrm{~s}$. Therefore, higher grasping force values were predicted to be obtained over a longer period of

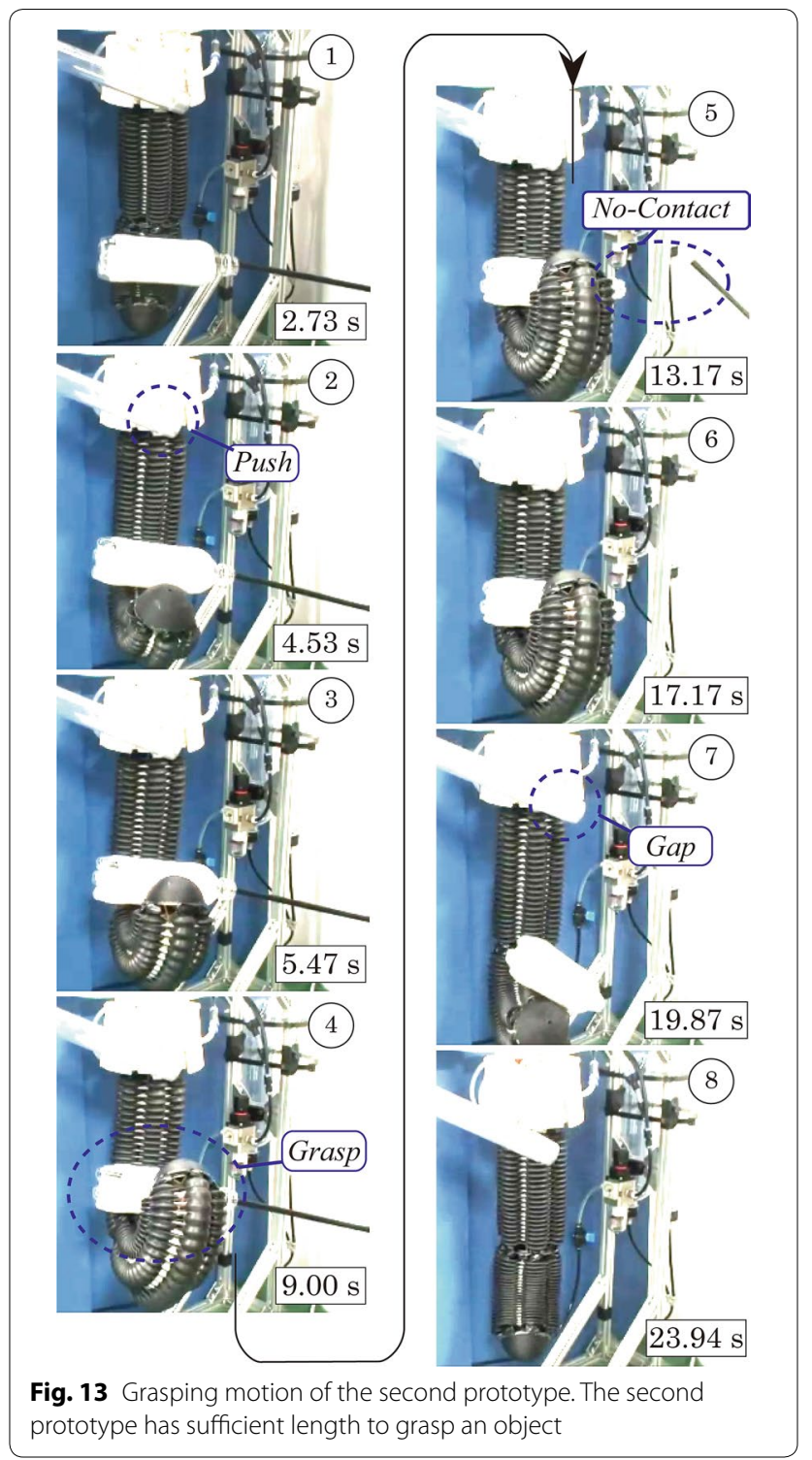

time. Moreover, the inner pressure becomes higher, the grasping force of the second prototype becomes bigger. The realization of the desired basic grasping performance (a higher pressure corresponded to a larger grasping force) was confirmed from this experiment, in which the grasping force over a period of $10 \mathrm{~s}$ was measured.

\section{Second prototype extended grasping part: influence of bending direction}

The grasping force in various directions (corresponding to a given "direction angle") was measured with the aim of ascertaining whether this configuration can provide an even grasping force in an omnidirectional manner. The direction angle was defined as shown in Fig. 16. The $30^{\circ}, 90^{\circ}$, and $150^{\circ}$ angles in the figure indicate that air was 


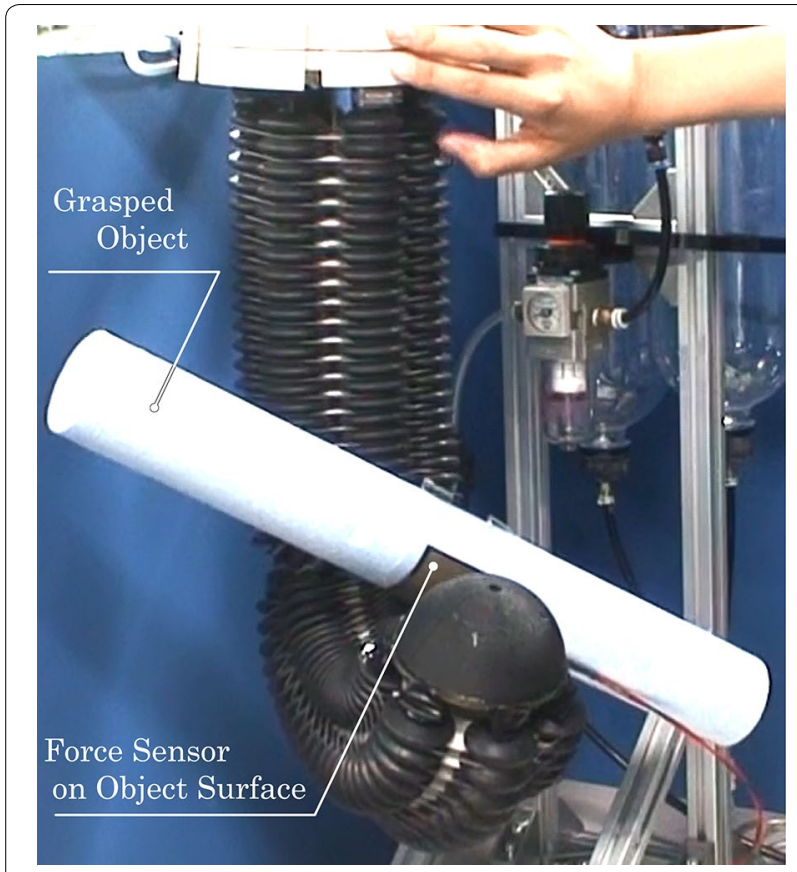

Fig. 14 Grasping experiment with force sensor. An object with a force sensor is grasped and the grasping force is measured for various pressures

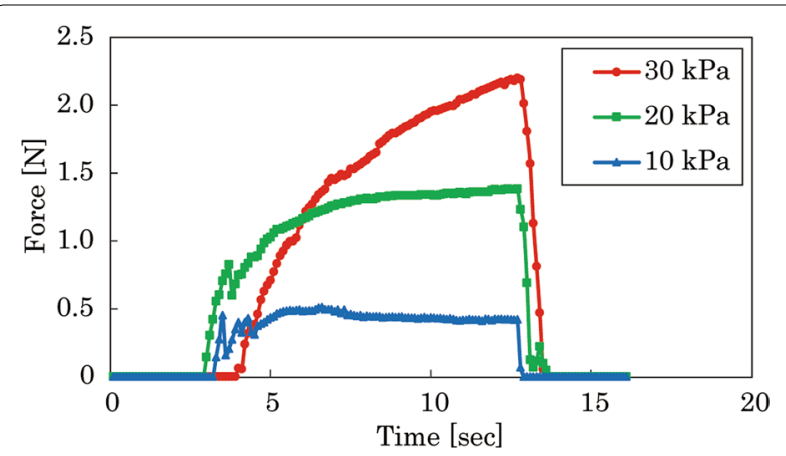

Fig. 15 Grasping force results for various applied pressures. The changes in the grasping force over time are shown for various applied pressures

supplied to one bellows at $30 \mathrm{kPa}$. Note that $0^{\circ}, 60^{\circ}, 120^{\circ}$, and $180^{\circ}$ angles indicate that two bellows are supplied with air. Therefore, a higher force was predicted to be obtained for a double-bellows operation compared to the single-bellows case. Accordingly, an object made of PET with a $70 \mathrm{~mm}$ diameter, $200 \mathrm{~mm}$ length, and $145 \mathrm{~g}$ weight was grasped for $10 \mathrm{~s}$. The average grasping force for the 9-10 s period was evaluated. The initial condition herein was fixed using a guide. Figure 17 shows the experimental setup, which depicts motion at $120^{\circ}$.

Figure 18 presents the results. As expected, higher forces than those for the $30^{\circ}, 90^{\circ}$, and $150^{\circ}$ angles were

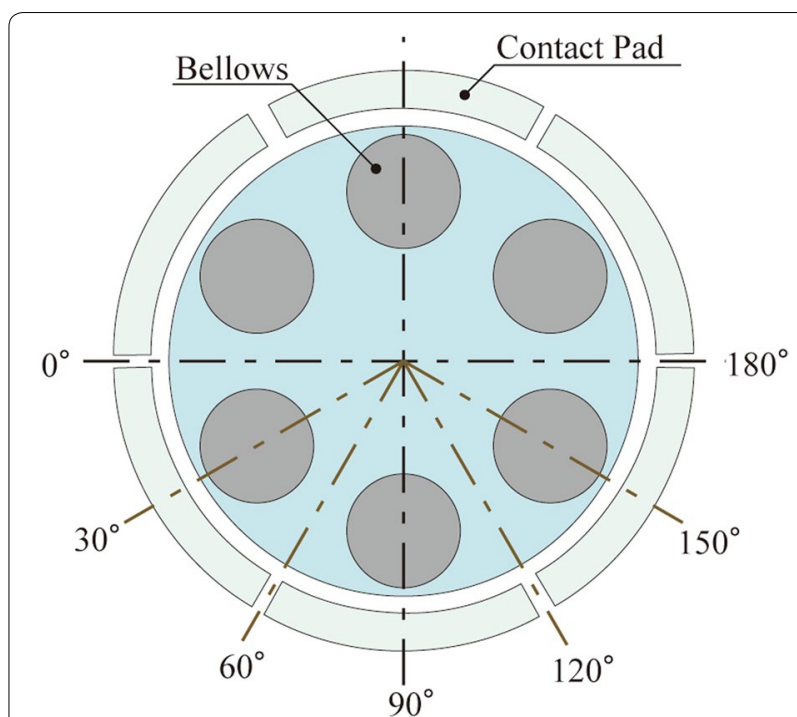

Fig. 16 Simplified cross-sectional view of prototype. The same setup is shown from above. The angles of $30^{\circ}, 90^{\circ}$, and $150^{\circ}$ mean that one bellows receives air. For $0^{\circ}, 60^{\circ}, 120^{\circ}$, and $180^{\circ}$, two bellows are supplied with air

measured for the $0^{\circ}, 60^{\circ}, 120^{\circ}$, and $180^{\circ}$ cases. The average for one bellows was $2.1 \mathrm{~N}$, while that for two bellows was $4.5 \mathrm{~N}$. The prototype could more stably grasp the object when two bellows were used because the contact was made at two points. Overall, we have confirmed that this configuration can apply similar forces in an omnidirectional manner even though differences were apparent between the single- and double-bellows cases. Increasing the resolution by increasing the air camber can reduce the difference between these forces.

\section{Second prototype extended grasping part: influence of gravity}

Subsequently, we experimentally observed the weight of the prototype. Observing the bending movement of the prototype with and without its own weight during $10 \mathrm{~s}$ with the contact pad was pushed in each situation. On the one hand, the top part of the hand is toward down in the presence of the object's own weight. On the other hand, the hand was positioned horizontally with respect to the direction of gravity without the object's own weight. We reduced the friction by placing the prototype on a stand equipped with a roller. The internal pressure used for the experimental conditions was $15 \mathrm{~Pa}$. The target object was a cylinder made of polystyrene foam with a $50 \mathrm{~mm}$ diameter, $400 \mathrm{~mm}$ length, and $145 \mathrm{~g}$ weight. The flow rate at the sensor (PFM550-01-1, SMC Corporation) was measured in each case. Figure 19 shows the experimental results. Figure 20 presents the measured flow rate. A larger curve was observed in the case where 

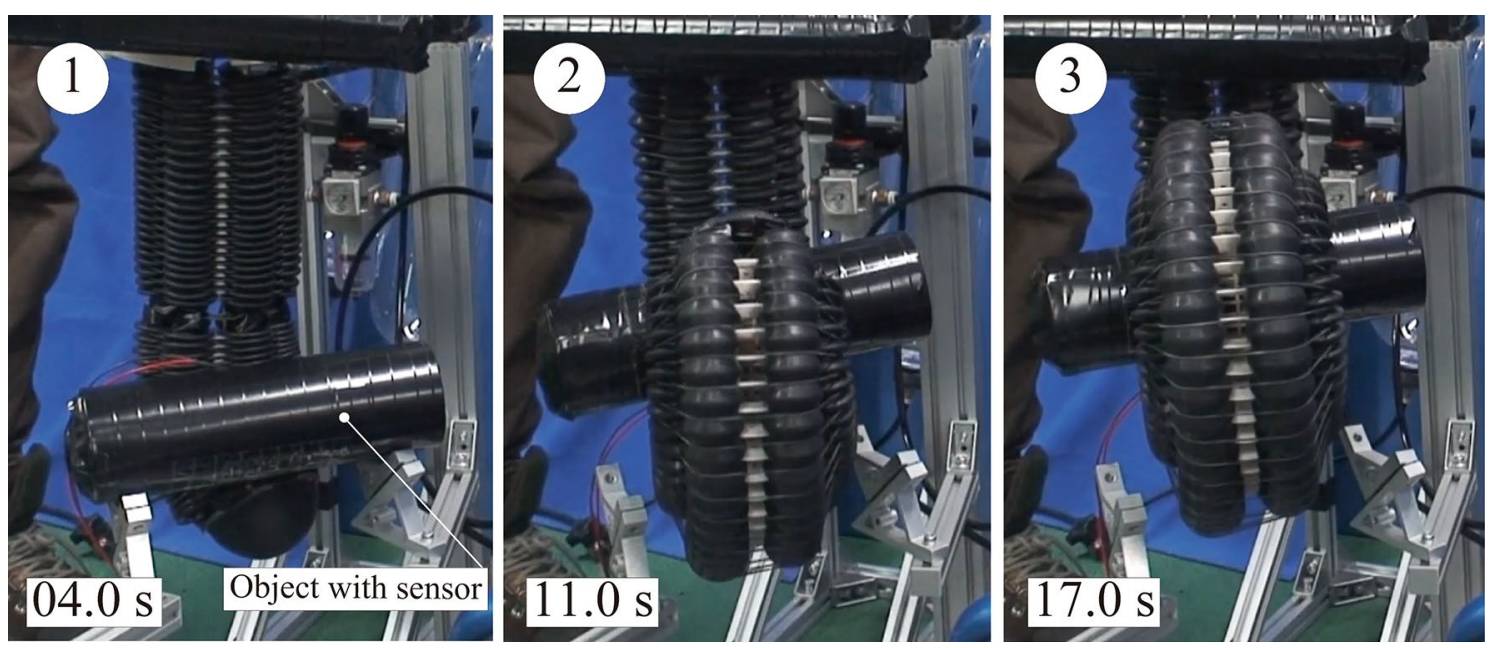

Fig. 17 Photograph of device for $120^{\circ}$ object position. An object with an attached force sensor makes contact with the prototype at an angle of $120^{\circ}$ from the initial position. The two bellows are supplied with air in response

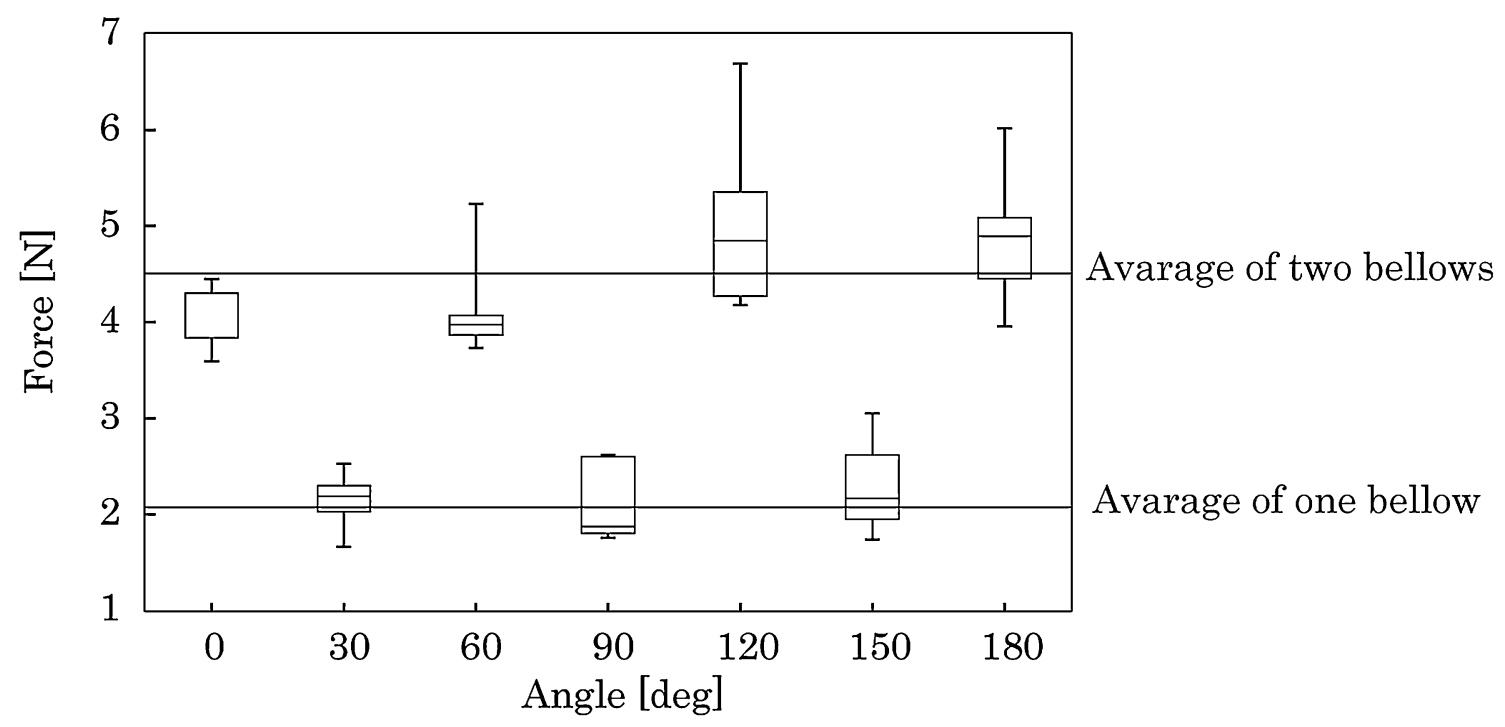

Fig. 18 Grasping force in various directions. The grasping force values for the angles given in Fig. 20

own weight was considered. As shown in (3) of Fig. 19b, the prototype pushed its own contact pad across the object after the large curve. In other words, it exhibited the object as self-holding. Furthermore, the flow meter in contact with the object for $2 \mathrm{~s}$ exhibited self-holding in the subsequent $3 \mathrm{~s}$. The result showed that own weight greatly influenced posture after the object was in contact, and should not be ignored. The curve became sufficiently large when its contact pad was pressed. This result experimentally proved that self-holding was possible. Help is needed during designing to build a theoretical value in light of own weight and face the future challenges.

\section{Conclusion}

This study proposed a mechanism for achieving reflexive omnidirectional bending in pneumatic continuum robots. The proposed mechanism has advantages for use as an explosion- and radiation-proof system because it need not be equipped with electronic components (e.g., solenoid valves, and sensors). A mechanical system based on a coupled drive mechanism with touch detection by mechanical port valves was devised, designed, and prototyped to realize the proposed design.

A successful realization of the proposed prototype configuration's fundamental characteristics was confirmed by 

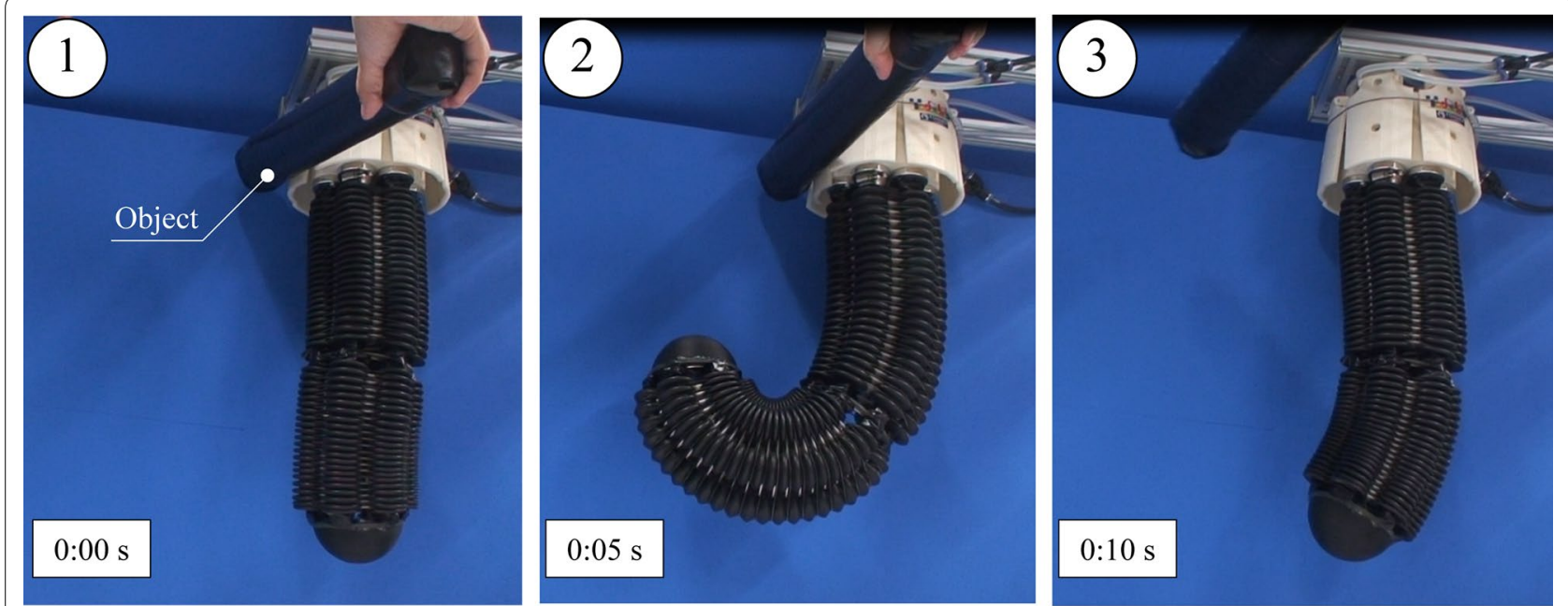

a Grasping motion with own-weight
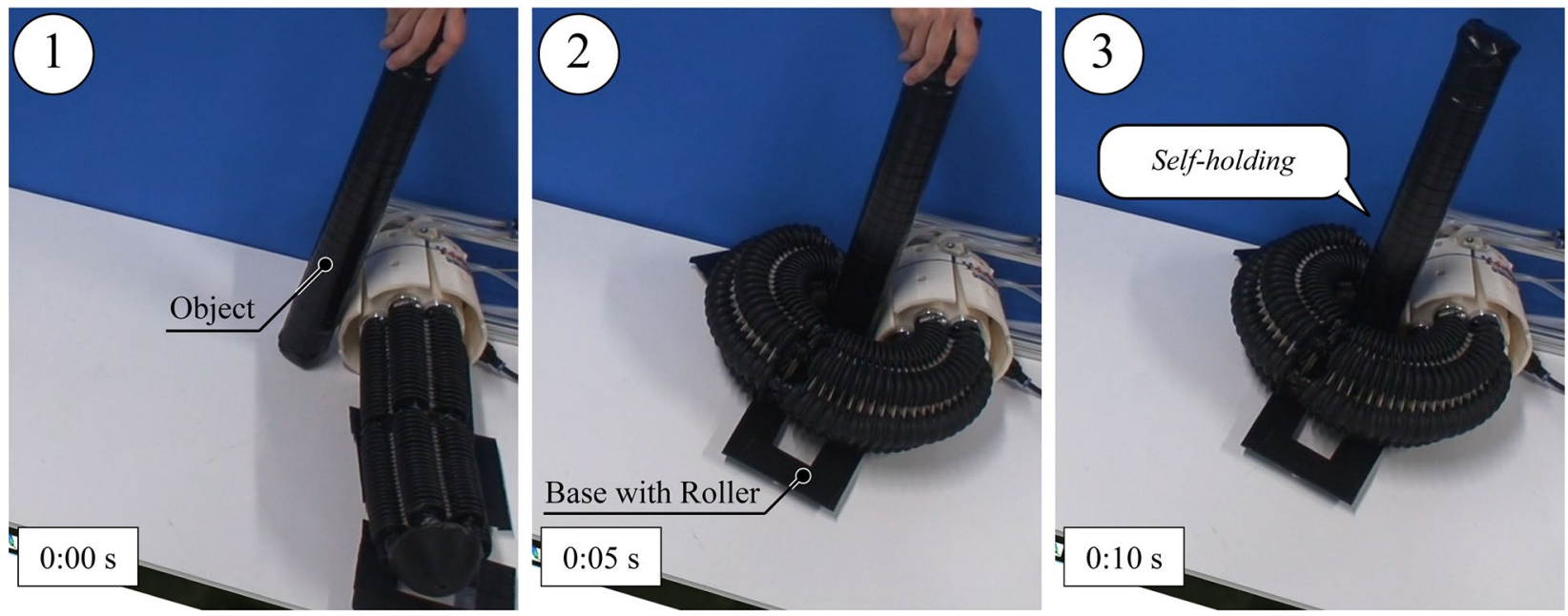

b Grasping motion without own-weight

Fig. 19 Grasping experiment with gravity and without own weight

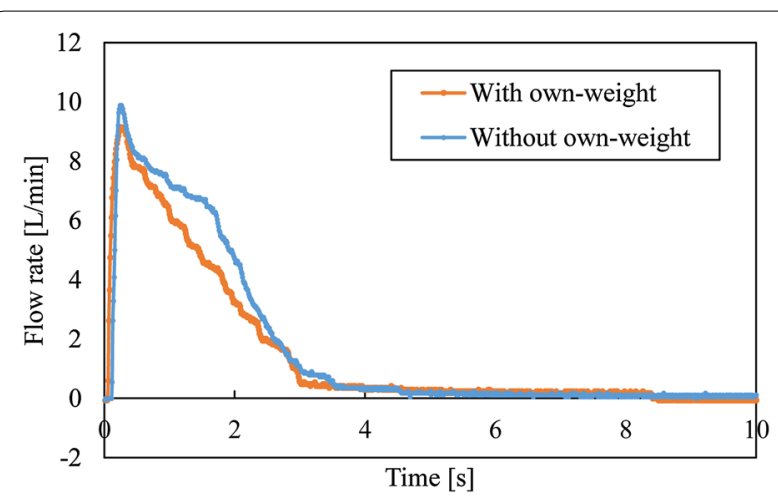

Fig. 20 Flow rate at the time of curve modeling and conducting first prototype experiments of posture and omnidirectional motion. A second prototype was also designed to improve the grasping performance. A quantitative evaluation of the resultant grasping force and the influence of bending direction and gravity was conducted via an experiment, which demonstrated the efficacy of the proposed mechanism. We confirm that the reflexive omnidirectional bending mechanism can be extended.

The proposed design will be further refined in the future. Greater stiffness will particularly be added to the mechanism to increase its grasping force $[18,19]$. Miniaturization, smaller-force triggering, and whole-body 
detection will be realized. Furthermore, a theoretical value in light of own weight must be built.

\section{Additional files}

Additional file 1. The movie confirm that this configuration can bend in an omnidirectional manner relative to the contact point.

Additional file 2. The second prototype is equipped to grasp an object.

\section{Authors' contributions}

ET and KT conceived the study, developed the device, carried out all experiments, analyzed data, and drafted the manuscript. TY, MK and ST participated in the research design and sequence alignment. All authors read and approved the final manuscript.

\section{Acknowledgements}

This study has been done as the part of the research program ImPACT: Tough Robotics Challenge.

\section{Competing interests}

The authors declare that they have no competing interests.

Received: 24 February 2016 Accepted: 4 November 2016

Published online: 17 November 2016

\section{References}

1. Walker ID (2013) Continuous backbone "Continuum" robot manipulators. ISRN Robot, Vol. 2013, Article ID 726506. http://www.hindawi.com/journals/isrn/2013/726506/. Accessed 30 Nov 2015

2. Hirose S (1987) Biologically inspired robots. Ind Comm, Japan

3. Mori M, Hirose S (2001) Development of active cord mechanism ACM-R3 with agile 3D mobility. In: Proceedings of the IEE/RSJ international conference on intelligent robots and systems, p 1552-1557

4. Erkmen I, Erkmen AM, Matsuno F, Chatterjee R, Kamegawa T (2002) Serpentine search robots in rescue operation. IEEE Robot Autom Mag 9(2):17

5. Ayers J, Wilbur C, Olcott C (2000) Lamprey Robot. In: Proceedings of the 1st international symposium on aqua bio-mechanisms, p 327-332

6. Nabil S, Kai X (2013) US Patent US20130090763A1
7. Hirose S (1986) Differential coupled mechanism and its application. Draft Design 21(10):362-366

8. Tadakuma K, Tadakuma R, Teshigawara S, Mizoguchi Y, Hasegawa H, Terada K, Takayama T, Omata T, Ming A, Shimojo M (2008) The morphing omnidirectional gripper "Morphing Omni-Gripper" with low melting point alloy. The 26th Annual Conference of The Robotics Society of Japan, 1E1-01

9. Bancon G, Huber B (1982) Depression and grippers with their possible applications. 12th ISIR, Paris, p 321-329

10. Brown E, Rodenberg N, Amend J, Mozeika A, Steltz E, Zakin MR, Lipson $\mathrm{H}$, Jaeger HM (2010) Universal robotic gripper based on the jamming of granular material. Proc Natl Acad Sci 107:18809-18814

11. Clement MG, Thierry L (1998) US Patent 5,762,390

12. Dollar AM, Howe RD (2010) The highly adaptive SDM hand: design and performance evaluation. Int J Robot Res 29(5):585-597

13. Rolf M, Steil JJ (2014) Efficient exploratory learning of inverse kinematics on a bionic elephant trunk. IEEE Trans Neural Netw Learn Syst 25:1147-1160

14. Suzumori K (1989) Flexible Microactuator (1st Report, Static Characteristics of 3 DOF Actuator). Trans Jpn Soc Mech Eng Ser C 55(518):2547-2552

15. Otherlab. https://otherlab.com/. Accessed 26 Jun 2015

16. McMahan W, Jones B A, Walker I D. (2005) Design and Implementation of a multi-section continuum robot: air-octor. In: Proceedings of the IEEE/RSJ international conference on intelligent robots and systems, $p$ 3345-3352

17. Tsukagoshi H, Kitagawa A, Segawa M (2001) Active hose: an artificial elephant's nose with maneuverability for rescue operation. In: Proceedings of the 2001 IEEE international conference on robotics \& automation, p 2454-2459

18. Kim YJ, Cheng S, Kim S, lagnemma K (2013) A novel layer jamming mechanism with tunable stiffness capability for minimally invasive surgery. IEEE Trans Rob 29:1031-1042

19. Cheng NG, Lobovsky MB, Keating SJ, Setapen AM, Gero KI, Hosoi AE, lagnemma KD (2012) Design and Analysis of a Robust, Low-cost, Highly Articulated Manipulator by Jamming of Granular Media. IEEE International Conference on Robotics and Automation, pp. 4328-4333

\section{Submit your manuscript to a SpringerOpen ${ }^{\circ}$ journal and benefit from:}

- Convenient online submission

- Rigorous peer review

- Immediate publication on acceptance

- Open access: articles freely available online

- High visibility within the field

- Retaining the copyright to your article 